\title{
Soldats et citoyens. Naissance du service militaire en France et en Prusse
}

\section{Annie Crépin}

\section{(2) OpenEdition \\ 1 Journals}

\section{Édition électronique}

URL : https://journals.openedition.org/ahrf/9543

DOI : 10.4000/ahrf.9543

ISSN : 1952-403X

Éditeur :

Armand Colin, Société des études robespierristes

\section{Édition imprimée}

Date de publication : 1 juin 2007

Pagination : 209-211

ISSN : 0003-4436

\section{Référence électronique}

Annie Crépin, «Soldats et citoyens. Naissance du service militaire en France et en Prusse », Annales

historiques de la Révolution française [En ligne], 348 | Avril-Juin 2007, mis en ligne le 23 juillet 2008,

consulté le 03 mai 2022. URL : http://journals.openedition.org/ahrf/9543 ; DOI : https://doi.org/

10.4000/ahrf.9543

Ce document a été généré automatiquement le 3 mai 2022.

Tous droits réservés 


\title{
Soldats et citoyens. Naissance du service militaire en France et en Prusse
}

\author{
Annie Crépin
}

\section{RÉFÉRENCE}

Thomas Hippler, Soldats et citoyens. Naissance du service militaire en France et en Prusse, préface d'Étienne Balibar, Paris, PUF, coll. Pratiques théoriques, 2006, 357 p., ISBN 2-13-053697-2, $29 €$.

1 Cet ouvrage est la version française de la thèse soutenue en anglais par Th. Hippler en 2002 à l'Institut européen de Florence et qu'il avait présentée dans le $n^{\circ} 3$ de 2003 des AHRF. L'auteur a une double formation d'historien et de philosophe et son œuvre est un nouveau témoignage de la fécondité pour l'histoire militaire des croisements entre disciplines.

Dans une introduction méthodologique qui est un modèle du genre, il explicite longuement les buts qu'il se propose. Sur le plan philosophique, il prend ses distances avec les conceptions foucaldiennes. Là où Foucault ne voit dans les institutions de l'État contemporain qu'un processus disciplinaire, Th. Hippler montre qu'à la différence de la prison, de l'asile ou de l'hôpital psychiatrique, le service militaire est une preuve de la complexité de la formation de cet État, plus « rusé » que son prédécesseur de l'époque moderne puisque, pour justifier la contrainte qu'il exerce, il fait jouer les ressorts de la volonté de l'individu et de la souveraineté populaire. La soumission n'entre pas en antagonisme avec la citoyenneté ni l'autonomie politique avec la discipline sociale. Au contraire, elles se renforcent mutuellement. Il est donc des contradictions fondatrices. C'est aussi à travers leur interaction qu'on peut réfléchir à l'évolution des concepts de peuple et de nation. Mais on ne peut manier ces concepts comme des abstractions, sans tenir compte de leur historicité. C'est donc une histoire culturelle que l'historien - et 
plus seulement le philosophe - Th. Hippler nous invite à lire et qui enveloppe à la fois les pratiques sociales et le sens de ces pratiques. La généalogie du service militaire est selon lui le lieu privilégié de l'étude du double mouvement de transformation des pratiques sociales et de construction sociale de leur sens historique. Ces considérations ont dicté le choix des sources et leur utilisation à trois niveaux de l'analyse, «macrosources » telles que discours officiels ou parlementaires et lois, sources subjectives, ainsi les écrits personnels, lettres, œuvres de fiction, à travers lesquelles on peut saisir l'expérience des soldats-citoyens et des conscrits, enfin "méta niveau » des textes littéraires et philosophiques qui abordent le sens de l'institution.

3 L'approche comparatiste elle même n'est comparatiste... qu'en apparence, car l'objet de l'étude est transnational. Pour l'auteur, les cas français et prussien ont constitué deux moments distincts et non deux modèles différents d'un même processus, d'où la structure du livre composé de deux parties, intitulées respectivement «Le moment français » et « Le moment prussien ». Chacun des quatre chapitres de la première partie répond terme à terme aux quatre chapitres de la seconde partie.

4 Suivant les degrés assignés par l'auteur à son analyse, le premier chapitre de la première partie est consacré à une approche historique. L'évolution du recrutement des forces armées est mise en parallèle avec la construction et la centralisation croissante de l'État absolutiste français. Pour retracer cette évolution, l'auteur utilise avec finesse les travaux classiques, tels ceux d'A. Corvisier, mais on peut regretter qu'il néglige les auteurs anglo-saxons comme S. Scott, quand il évoque la fin de l'Ancien Régime et, anticipant sur le second chapitre, les débats intellectuels que suscitèrent alors la nature et le rôle de l'armée. Le second chapitre, plus philosophique (encore que l'auteur, dans un constant va-et-vient, ne sépare jamais complètement les deux approches), étudie la réinterprétation par les Lumières de l'obligation militaire dans l'optique de la vertu citoyenne et même de la construction du peuple et de la communauté sociale. Les contradictions qui sont à la base de la conscription «[...] qui nie ce qu'elle affirme et soutient ce qu'elle contredit » (p. 11) sont déjà toutes entières dans l'œuvre des philosophes et surtout de Rousseau dans la pensée duquel le service militaire joue un rôle crucial. La réflexion de Mably, celle des écrivains militaires, y compris ceux qui sont partisans de la réaction nobiliaire comme le chevalier d'Arcq, sont minutieusement étudiées. Il aurait été pertinent cependant que l'auteur se montre plus nuancé à propos de Guibert car, selon nous, celui-ci souhaitait des soldats-citoyens mais pas nécessairement que chaque citoyen soit soldat. Le troisième chapitre montre « en situation » comment la Révolution fait du service militaire à la fois l'expression la plus haute des droits civiques et une institution disciplinaire. "L'esclavage militaire » se transforme en pilier de la liberté politique, en concomitance avec la naissance de l'État contemporain. L'armée permanente devient l'émanation de la liberté, de la justice et de la raison. Ces extraordinaires constructions idéologiques, et même ce renversement conceptuel, qui tentent de surmonter les tensions entre citoyenneté et discipline et faire de l'armée l'incarnation du peuple souverain en même temps que le service militaire construit ce peuple par un processus d'autocréation, l'auteur les confronte d'une part aux projets de code militaire, à propos desquels il a des pages éclairantes, d'autre part aux perceptions qu'en ont les soldats et qui prouvent que de 1791 à 1793 ces constructions ont fonctionné. On regrette que le récent ouvrage d'A. Forrest, Napoleon's Men, ne soit pas exploité, du moins cité. Mais, et c'est l'objet du quatrième chapitre, les catégories identitaires de classe, de nation, de genre qu'on avait cru sublimées dans un idéal d'universel civique quelque peu abstrait, opposant la série 
de concepts vice-désordre-femelle-populace à la série vertu-ordre-homme-peuple, resurgissent sur d'autres plans. L'intégration induit de nouvelles exclusions. Au point de vue militaire, la dichotomie réapparaît entre l'obéissance passive et la discipline librement consentie, au niveau national, le concept de peuple englobe ceux qui sont sujets politiques et ceux et celles qui sont exclus de la sphère politique, au niveau international, le peuple érigé en nation unique se définit désormais en contraste avec les autres nations. Au niveau individuel enfin, alors que le citoyen-soldat est censé réaliser la synthèse de l'universel et du particulier, c'est dans son esprit que la sociabilité civique s'affronte avec les forces de l'intérêt et de l'égoïsme et cet affrontement est le plus tragique car il ne peut être résolu que par la mort du protagoniste. Il y a des pages très fortes sur la mort des combattants qui réalise l'égalité parfaite et représente le sommet éthique de la participation démocratique du citoyen à la respublica.

5 La seconde partie s'ouvre par un chapitre qui est le pendant du chapitre initial : il trace un parallèle entre l'histoire de la centralisation étatique en Prusse et celle de l'obligation militaire au sein du Kantonsystem. Au passage, Th. Hippler remet en question quelques idées reçues en faisant remarquer que le système cantonal est un aspect de l'absolutisme en Prusse, non le reflet des intérêts des hobereaux au détriment desquels il se développe. Comme chez les militaires philosophes français, un tel système donna lieu chez certains de leurs homologues germaniques à une réflexion intellectuelle, que l'auteur analyse avant de se livrer dans le sixième chapitre à une approche philosophique des conséquences idéologiques des défaites de la Prusse et de l'appropriation intellectuelle de la Révolution Française par l'idéalisme allemand, celui de Fichte et Kant, mais aussi par les romans philosophico-narratifs, ceux de Goethe notamment, et par le théâtre national, dont celui de Schiller. Ces penseurs se heurtent eux aussi aux contradictions entre la liberté et la contrainte, l'universel et le particulier : le service militaire et «l'humiliation héroïque » à laquelle il conduit, c'està-dire l'immolation des intérêts de l'individu au devoir du citoyen vertueux, est une façon de sublimer ces contradictions.

6 Paradoxalement, les implications de la Révolution furent conceptualisées par les intellectuels allemands davantage que par leurs contemporains français, ce qui permit à la bureaucratie réformatrice de s'inspirer directement des premiers qui lui fournissaient un cadre théorique tout fait. Les deux derniers chapitres de cette partie sont donc consacrés aux réformes prussiennes, au premier chef la réforme militaire, dont Th. Hippler dit qu'elles sont directement le résultat de la réception allemande de la Révolution française, et aussi aux avatars de la guerre de libération, à l'établissement de la landwehr et à ce qu'en dirent leurs protagonistes. Dans l'argumentation de la Commission de réorganisation militaire l'auteur voit la preuve de ce qu'il avançait précédemment: c'est une philosophie de l'histoire qui, avec la révolution kantienne dans le domaine de la pensée et la Révolution française en matière politique, rendit possible d'unir les contraires; on ne le voit nulle part plus que dans l'armée qui fonctionne comme médiation théorique et pratique de concepts qui paraissaient irréductiblement antagonistes. En Prusse comme en France, le service militaire devient une institution autocréatrice du peuple et de la nation, une institution éducative « totalisante » (avec des virtualités « totalitaires » que l'auteur décèle avec lucidité bien qu'il ne tombe pas dans la vulgate à la mode). On retrouve aussi les beaux passages de la première partie à travers l'évocation de la mise à mort symbolique de l'amour de soi du 
conscrit censée préparer sa "résurrection» en tant qu'être pleinement moral et anticipant sa mise à mort réelle sur le champ de bataille.

7 Ce dense et bel ouvrage s'achève sur une bibliographie franco-allemande très fournie où l'on regrette toutefois l'absence déjà déplorée plus haut des historiens anglo-saxons, A. Forrest et S. Scott, et aussi celle d'I. Woloch. 